\title{
Cardiology
}

\section{Synchronization of Wave Flows of Arterial and Venous Blood and Phases of the Cardiac Cycle: Part 3}

\author{
Alexander G. Kruglov, PhD, ScD; Valery N. Utkin; Alexander Yu. Vasilyev, PhD, ScD; \\ Andrey A. Kruglov, PhD \\ Central Research Institute of Radiation Diagnosis \\ Moscow, the Russian Federation
}

\begin{abstract}
Hemodynamic indices studied in healthy people were obtained by catheterization in various vascular areas: the chambers of the heart (ventricles, atria, coronary sinus), pulmonary trunk, sigmoid sinus, aorta, inferior vena cava, superior vena cava (SVC), and right hepatic vein (RHV). Using the mean values of the hemodynamic parameters, we constructed graphics of the curves of the central, arterial, and venous pressure, synchronized with each other, with an ECG, and with pulse wave.

The complex of hemodynamic curves, supplemented by curves of RHV and SVC, revealed a coincidence in one zone of temporal equalization of pressure (ZTEP) for values of the left atrium, coronary sinus, right ventricle, sigmoid sinus, SVC, and RHV(wedged). We identified this ZTEP as a hemodynamic trigger (T-point), assuming that this point is the trigger point for the launch of the high-energy processes of the right heart, forming the following cardiac cycle: the beginning of the systole of right ventricle, the opening of pulmonary valve. (International Journal of Biomedicine. 2018;8(4):288-291.)
\end{abstract}

Key Words: cardiac cycle $\bullet$ hemodynamic parameters $\bullet$ ECG $\bullet$ wave flows

\section{Abbreviations}

Ao, aorta; AV, aortic valve; CC, cardiac cycle; CS, coronary sinus; CNS, central nervous system; DP, diastolic pressure; IVC, inferior vena cava; IHH, intrahepatic hemodynamics; LA, left atrium; LV, left ventricle; MV, mitral valve; PC, pulmonary circulation; PT, pulmonary trunk; Pvs, pulmonary veins; PV, pulmonary valve; PW, pulse wave; RV, right ventricle; RA, right atrium; RHV, right hepatic vein; SC, systemic circulation; SP, systolic pressure SS, sigmoid sinus; SVC, superior vena cava; TV, tricuspid valve; ZTEP, zone of temporal equalization of pressure.

\section{Basic Part}

The purpose of this part of the work was to study the hemodynamic processes of RA, as a single hydrohemodynamic formation, including SVC and the mouth of RHV.

\section{Methods and Discussion}

In addition to the hemodynamic data given in previous articles, ${ }^{(1-4)}$ Table of Norms (Table 1) includes graphical curves: SVC and RHV(wedged, i.e., IHH).

*Corresponding author: Alexander G. Kruglov, PhD, ScD. Central Research Institute of Radiation Diagnosis. Moscow, the Russian Federation.E-mail: krag48@mail.ru
The hemodynamic contour of SVC almost completely repeats the hemodynamic contour of RA, making up a single hydrodynamic cavity with it (note the smaller dependence of the RA cavity on external factors: for example, breathing). The recording of hemodynamic curves during catheterization was carried out with the subject in the supine position (minimizing the effect of the gravity vector on the capacitive vessels), with holding the breath on the exhale (minimizing the dynamics of intrathoracic pressure on the capacitive vessels).

RHV(wedged), which we included in the graphic complex, demonstrates the dynamics of pressure in the venous part of the microcirculation vessels of the liver: IHHs.p. transmission pressure from Ao through the a.hepatica system; IHHd.p. - a pressure in the microcirculation system of the 
liver after a vascular response of arterioles to PW (according to the Ostroumov-Beilis reaction); straight sections of the graph reflect the pressure in the microcirculation system caused by pressure in the v.portae. Thus, the graphic representation of $\mathrm{IHH}$, in our opinion, reflects a number of functions that form the microcirculatory regulation of the liver, with the participation of both a.hepatica and v.portae. The combination of the CS impulse at the onset of RA systole and the suction effect of the systole itself stimulate the opening of outflow vessels from the liver and the inflow into it from v.portae. The myogenic response of arterioles restricts the a.hepatica inflow (and, we also assume, the v.portae inflow), which maintains a constant level of pressure in the liver acinus. Evidence of this is the constancy of the portal part of the graph of the IHH curve (despite the changing conditions of inflow and outflow associated with the activity of the heart), ensuring the autonomy of portal and acinus pressure, which contributes to the stabilization of biochemical processes in the liver acinus. It should be noted that the maximum number of graphic combinations in one ZTEP falls on the curves of IHH and SS, which, in our opinion, may indicate a combined effect of these zones (brain and splanchnic organs) on the formation of the cardiac cycle phases.

The coincidence of the successive spread of contractions of various parts of the heart with the course of Purkinje fibers was noted in early studies. ${ }^{(5)}$ In the embryonic heart, at the stage of development of the tube, which has already begun to pulsate, the venous sinus is first contracted, then successively the rudiments of auricles, ventricles, and aortic bulb. This is a period when the heart rudiments do not receive any nerve impulses (nerve fibers have not yet grown to muscle tissue); therefore, the impulse starts inside the organ, in its tissues, in particular, in the tissues of the venous sinus, spreading throughout the heart rudiment. At later stages of development, as well as in adult organisms, it was found that the impulse to contraction arises precisely in the part developing from the venous sinus (i.e., in the place of transition of SVC into RA (sinus node) and on the right triangle of the central fibrous body (atrioventricular node). Thus, the heartbeats, starting from the embryonic period and ending with the developed heart, are autonomous, having a myogenic nature. It is this system that forms the functional integrity of the heart, with the subsequent increasing role of CNS impulses in the heart rhythm when conscious, emotionally significant, stressful situations occur.

The fact of active, uninterrupted work of the denervated heart ${ }^{(6,7)}$ (i.e., in the absence of a direct connection with CNS) is evidence of the systemic autonomy of the heart and the cardiovascular system as a whole. A pulsed trigger is the electrical discharge of the sinus node, which triggers synchronized successive phases of the heart's activity, which is a hydrohemodynamic system consisting of the periodically connecting-disconnecting chambers and conducting vessels (separated by valves), the phase synchronization of which occurs according to the principle of overpressure at the inlet (into the vessel, into the chamber), with a feedback control system. Thus, a developed heart has an autonomous (biologically earlier, preceding nervous regulation) system for triggering a contraction of syncytium (having an automated nature), as well as a system of control signals from CNS, providing an adequate physiological response to environmental stimuli.

Control signals from CNS (n.vagus and sympathetic nerves) provide an adequate response of the heart (frequency, filling, etc.) to stimuli imposed by the external environment, and regulate the activity of the heart and adequate organ blood flow, providing a hemodynamic and metabolic response to environmental stimuli, if necessary (psychological image of danger, emotional stress, etc.).

For the figurative (complementary two-dimensional graphic picture) representation of the activity of the cardiovascular system, the synchronous interaction of the totality of vessels and chambers, which we graphically show in Table 1, we describe the hydrohemodynamics of the heart as a system as a whole. In the three-chamber block of ventricles operating in a quantum generator mode, the right and left ventricles (separated by an interventricular septum) form three pairs of successively alternating arterial and venous volumetric modules of variable capacity:

A) In diastole, the atrio-ventricular module is formed, as a result of combining the three-chamber block of ventricles with the atrial block at its complete separation from the aortopulmonary block. At the same time, the venous volumetric modules of variable capacitance of SC (IVC-SVC-RA-CS-RV) and PC (Pvs-LA-LV) are separated from arterial volumetric modules of variable capacitance of $\mathrm{SC}(\mathrm{Ao})$ and $\mathrm{PC}(\mathrm{PT})$ by the membrane valves at the outlet of the heart (with PV for SC and $\mathrm{AV}$ for $\mathrm{PC}$ ), separating them from the aorto-pulmonary block. In this phase, with open atrioventricular valves the atrioventricular module of the variable capacitance of SC and PC (the three-chamber block of ventricles + the atrial block) acts as a receiver of hemodynamic volumes and wave signals entering the right heart from the IVC and SVC exchange zones, and the left heart from the lung.

B) In the isometric phase of ventricular contraction with the closed heart valves, an atrial-spongy module of variable capacity is formed by connecting to the atrial block, the venous (spongy) myocardial chamber (by CS-bypass) when completely separated from the aorto-pulmonary block. At the same time, the venous volumetric modules of SC (IVCSVC-RA-CS) and PC (PV and LA) are separated from arterial volumetric modules of $\mathrm{SC}(\mathrm{Ao})$ and $\mathrm{PC}(\mathrm{PT})$ by two membranes at the entrance and exit from the ventricular cavities (TV and PV for SC and PC, MV and valve AV for PC and SC). In this phase, with the participation of both ventricles, a CS impulse is formed and generated (in the initial phase of LV: Asd1 period), complemented by RV (Ss1 period). Unlike CS, whose impulse enters RA during formation, the spheroid of pulse wave enters the bloodstream already formed during the entire isometric ventricular contraction phase, as a result of the opening of the semilunar valves.

C) In the systole, a ventricle-aortic-pulmonary module is formed by combining the three-chamber block of ventricles with the aorto-pulmonary block (ventricular- aorto - pulmonary module), having a hemodynamic connection with the atrial block by CS (supporting pressure of right atrium as feedback). 




\begin{tabular}{|c|c|}
\hline \multicolumn{2}{|c|}{ Abbreviations } \\
\hline \begin{tabular}{|l|} 
I0 \\
- characteristic points of PW (V.V. Boronoev)
\end{tabular} & $\mathrm{H}(\mathrm{PV})-$ closing of PV \\
\hline $\mathbf{A}(\mathbf{Q})$ - isometric ventricular relaxation & $G(T V)$ - opening of TV \\
\hline$B(Q)$ - actual ventricular diastole & $\mathrm{H}(\mathrm{AV})-$ closing of $\mathrm{AV}$ \\
\hline C(LV) - LV diastole & G(MV)- opening of MV \\
\hline$A(L V)$ - isometric relaxation of $\mathrm{LV}$ & $\mathrm{H}(\mathrm{TV})-$ closing of TV \\
\hline B(LV)- actual LV diastole & $\mathrm{H}(\mathrm{MV})$ - closing of MV \\
\hline C(RV)- RV diastole & G(PV)- opening of PV \\
\hline$A(R V)$ - isometric relaxation of $R V$ & $H(P V)-$ closing of PV \\
\hline$B(R V)-$ actual RV diastole & Asd1- asynchronous period of ventricular systole-diastole -1 \\
\hline$D(Q)$ - isometric ventricular contraction & Ss1- synchronization period of isometric ventricular contraction-1 \\
\hline E(Q) - actual ventricular systole & As1- asynchronous period of ventricular systole -1 \\
\hline F(LV)- LV systole & Ss2- synchronization of the actual ventricular systole -2 \\
\hline$D(L V)-L V$ isometric ventricular contraction & Asd2- asynchronous period of ventricular systole-diastole -2 \\
\hline E(LV)- actual LV systole & Ad1- asynchronous period of ventricular diastole -1 \\
\hline F(RV)- RV systole & Sd1- period of synchronization of ventricular diastole-1 \\
\hline $\mathbf{D}(\mathbf{R V})-\mathrm{RV}$ isometric contraction period & ロ - dynamic curve ZTEP \\
\hline E(RV)- actual RV systole & $\leftrightarrow-\mathrm{PW}$ phases and $\mathrm{O}-$ coincidence between $\mathrm{ZTEP}$ and $\mathrm{PW}$ points \\
\hline G(AV)- opening of AV & O- T-point \\
\hline
\end{tabular}


At the same time, the venous volumetric modules of SC (IVCSVC-RA-CS) and PC (Pvs-LA) are separated from the arterial volumetric modules of SC (LV-Ao) and PC (RV-PT) by a single membrane at the entrance to the three-chamber block of ventricles (TV for SC and MV for PC).

We believe that the described change of temporary arterial and venous volumetric modules of variable capacity is carried out due to the movement of hemodynamic volumes by the myocardium and the synchronized creation of excessive hydrodynamic pressure on the inflow to the valves of the heart vessels and chambers. The combination of "closing-opening" valves is implemented with the participation of fibrous filaments, which are an integral part of the fibrous skeleton of the heart, penetrating the entire myocardium. We believe that the wave impulses generated by the myocardium propagate both through incompressible blood boles and through the system of the fibrous skeleton of the heart, carrying out synchronous autoregulation of the heart, which does not depend on CNS.

We believe it is extremely important to note the coincidence of the values of hemodynamic "curves" in one ZTEP(LA-CS-SVC-RV-RHV-SS) that coincides with the opening of $\mathrm{PV}$, synchronized (vertically) with the minimum pressure level in Ao. We designated this ZTEP as a hemodynamic trigger (T-point), assuming that this phase is the trigger point for the launch of the high-energy processes of the right heart, which form the following CC: the beginning of RV systole, the opening of PV (Table 1). Changes in the conditions of the body's functioning (changing the body's position relative to the gravity vector, etc.) can lead to the migration of the T-point, we believe, in the direction of lower pressure levels (i.e., in RA) that can create combinations of hemodynamic participants of ZTEPs, different from those registered by us.

In conclusion, concurrence of the values of hemodynamic curves (1. LA, 2. CS; 3. SVC; 4. RV; 5. RHV; 6. SS) in one ZTEP, synchronous with the opening of PV and the minimum pressure level in Ao, for the conditions in which human catheterization was carried out, is a trigger hemodynamic point (T-point) - launch point - of high-energy processes in the right heart, which form the following CC: the beginning of RV systole, the opening of PV. We believe that a change in the body's position relative to the gravity vector can lead to the migration of the T-point, which can create combinations of the structure of ZTEPs different from those registered by us.

\section{Conflict of interest}

The authors declare that they have no competing interests.

\section{References}

1. Gebel GYa, Kruglov AG, Utkin VN, Bagdatyev VE, Dasaev AN, Golostenova LM. [On the role of the coronary sinus of the human heart in the norm (regulation of a number of functions to the issue of synchronization in the circulatory system)]. Proceedings of the 10th Conference on Space Biology and Aerospace Medicine. M., 1994:56-57. [Article in Russian].

2. Kruglov AG, Utkin VN, Vasilyev AY, Sherman VA. Human Homeostatic Control Matrix in Norm. International Journal of Biomedicine. 2016;6(3):184-9.

3. Kruglov AG, Utkin VN, Vasilyev AY. Synchronization of Wave Flows of Arterial and Venous Blood with Phases of the Cardiac Cycle in Norm: Part 1. International Journal of Biomedicine. 2018;8(2):123-128.

4. Kruglov AG, Utkin VN, Vasilyev AY, Kruglov AA. Synchronization of Wave Flows of Arterial and Venous Blood and Phases of the Cardiac Cycle with the Structure of the Peripheral Pulse Wave in Norm: Part 2. International Journal of Biomedicine. 2018;8(3):177-181.

5. Zavarzin AA, Rumyantsev AA. Course of Histology. 6th edition. M. Medgiz; 1946.

6. Donald DE, Shepherd JT. Initial Cardiovascular adjustment to exercise in dogs with chronic cardiac denervation. Am J Physiol. 1964; 207:1325-9.

7. Donald DE, Shepherd JT. Sustained capacity for exercise in dogs after complete cardiac denervation. Am J Cardiol. 1964;14:853-9. 